\title{
Mesure de la charge de travail en pharmacie hospitalière : analyse descriptive des données d'un établissement de santé québécois de 2004 à 2013
}

\author{
Sophie Dubois, Denis Lebel et Jean-François Bussières
}

\section{INTRODUCTION}

T a consignation des activités professionnelles est une des _composantes importantes de l'exercice optimal de toute profession. Elle permet notamment la communication entre professionnels, la continuité des soins au fil du temps, la détection des problèmes cliniques et administratifs et l'évaluation périodique des pratiques.

La consignation des activités professionnelles peut cibler différentes actions au moyen de divers outils. Par exemple, un professionnel peut inscrire au dossier pharmacologique informatisé un objectif thérapeutique qu'il souhaite atteindre avec le patient dans le cadre de la surveillance de la thérapie médicamenteuse ou encore inscrire au dossier patient une intervention qu'il souhaite effectuer auprès d'un professionnel.

Plusieurs sociétés savantes reconnaissent l'importance de la consignation des activités pharmaceutiques. L'Ordre des pharmaciens du Québec (OPQ) définit la consignation comme étant "l'inscription aux dossiers de toutes les informations influençant l'évaluation pharmacothérapeutique des dossiers patients $"$. En 2003, l'American Society of Health-System Pharmacists a publié des lignes directrices sur la consignation, lesquelles ont été mises à jour en $2008^{2}$. La Société française de pharmacie clinique a développé un outil de valorisation des activités pharmaceutiques afin de permettre aux pharmaciens hospitaliers de recueillir les interventions de pharmacie clinique, de les quantifier et de communiquer des données dans le but d'uniformiser les pratiques ${ }^{3}$. L'OPQ exige dans son programme d'inspection professionnelle périodique que le pharmacien consigne au dossier du patient tous les renseignements essentiels, ses interventions ainsi que ses notes de suivi ${ }^{1}$.

Afin d'assurer le bon usage des ressources professionnelles en établissement de santé, plusieurs initiatives ont été mises en place pour décrire et suivre sur écran la consignation des activités pharmaceutiques. Au début des années quatre-vingt, un système de mesure de la charge de travail en pharmacie a été mis en place avec la contribution de Statistiques Canada ${ }^{4}$. Les améliorations qui ont été apportées à la démarche au cours des années suivantes ont permis de préciser les indicateurs et de proposer des outils de collecte aux pharmaciens et au personnel administratif ${ }^{5-7}$. Bien que ces démarches aient mené à l'adoption d'un système de mesure de la charge de travail pharmaceutique dans certaines provinces (p. ex. Saskatchewan, Ontario), ces systèmes ont été rapidement abandonnés ${ }^{8}$. Les travaux qui ont été menés au cours des années deux mille ont contribué au développement d'outils électroniques intégrés ou non aux systèmes d'information de la pharmacie ${ }^{9,10}$.

Plus récemment, trois groupes de recherche se sont intéressés à la détermination d'indicateurs de performance visant à décrire et à comparer les activités de soins pharmaceutiques. En utilisant une technique de type Delphi, les équipes de Magarinos-Torres et coll. au Brésil $(2007)^{11}$, de $\mathrm{Ng}$ et coll. en Nouvelle Zélande $(2010)^{12}$, et de Fernandes et coll. au Canada $(2015)^{13}$ ont déterminé respectivement quatre, dix et huit indicateurs clés de performance de la pratique pharmaceutique. Les travaux du groupe canadien ont notamment permis la publication d'un guide de formation, et d'autres travaux seront menés afin d'encourager la comptabilisation de ces indicateurs pour décrire la charge de travail des pharmaciens.

Au Québec, il n'existe pas de directives administratives encadrant la mesure de la charge de travail en pharmacie. Selon le manuel de gestion financière du ministère de la Santé et des Services sociaux du Québec (MSSS), seules les dépenses en médicaments et fournitures (composante médicament) et en ressources humaines (composante professionnelle) sont prises en compte pour l'établissement des ratios de performance 
relatifs aux activités visant les patients hospitalisés (p. ex. nombre d'heures travaillées ou rémunérées par admission). L'unité de mesure des activités ambulatoires (nombre de visites de patients) ne sert pas à établir le ratio de performance.

En dépit de ces directives limitées, dès 1998, le département de pharmacie du Centre hospitalier universitaire (CHU) SainteJustine s'est doté d'un journal de bord des pharmaciens en version papier, afin de consigner la pratique pharmaceutique. En 2003, une version électronique de ce journal a été intégrée à l'intranet de l'établissement. L'outil a été développé, préalablement testé, puis implanté sur la base d'une revue documentaire initiale. Les changements apportés au fil du temps visent à préciser les définitions de certains indicateurs, à améliorer l'ergonomie de l'outil et à optimiser le temps de consignation. L'outil, qui est utilisé depuis son introduction, permet de décrire la charge de travail de l'ensemble des activités cliniques des pharmaciens.

À la suite des travaux de Fernandes et coll. et en vue d'appliquer ces nouveaux indicateurs de performance, nous nous sommes intéressés à l'analyse des données recueillies dans le journal de bord des pharmaciens de notre établissement sur une période de dix ans ${ }^{13}$.

\section{MÉTHODE}

Il s'agit d'une étude descriptive et rétrospective.

L'objectif principal vise à décrire le profil des activités pharmaceutiques au sein d'un établissement de santé sur la base d'un journal de bord quotidien. L'objectif secondaire est de présenter et de comparer des indicateurs de performance utilisés pour la gestion du département.

L'étude se déroule au CHU Sainte-Justine, un centre hospitalier universitaire mère-enfant de 500 lits. Le département de pharmacie comporte 36 équivalents temps plein pharmaciens. Le département de pharmacie offre des services pharmaceutiques à raison de 104 heures par semaine et des soins pharmaceutiques décentralisés au sein de 30 programmes de soins hospitaliers et de 28 programmes de soins ambulatoires. Dans notre établissement, les services pharmaceutiques correspondent notamment à la validation des ordonnances de médicaments avant qu'ils soient distribués dans les unités et administrés aux patients, à la validation des préparations magistrales et des doses de suspension orale préparées en seringues individuelles pour la pédiatrie.

L'étude porte sur une période de 10 années civiles consécutives. Les données qui ont été extraites de la base de données des activités pharmaceutiques couvrent la période du $1^{\mathrm{er}}$ janvier 2004 au 31 décembre 2013. La période ciblée se justifie par le nombre d'années prises en compte (c.-̀̀-d. au moins 10 ans de recul) pendant lesquelles le journal de bord a subi peu de modifications. À partir de 2014, des ajustements à ce journal ont été nécessaires en raison des nouveaux indicateurs clés de performance en pharmacie clinique, ce qui limite la comparabilité des données. L'établissement du profil quantitatif des activités a eu lieu à partir des données brutes saisies par chaque pharmacien selon sa fonction à l'horaire. L'organisation des activités pharmaceutiques en place prévoit de 20 à 25 fonctions à l'horaire de travail quotidien. Depuis 2003, chaque pharmacien tient régulièrement le journal de bord sur l'intranet.

Le journal de bord a permis de calculer et d'analyser les variables suivantes : 1 ) nombre d'heures travaillées en temps pharmacien selon les cinq axes de pratique (gestion, services, soins, enseignement, recherche), 2) nombre de demandes d'information, 3) nombre d'interventions pharmaceutiques selon le type, 4) nombre de patients suivis en externe et en hospitalisation, 5) nombre de jours-présence d'étudiants de $1^{\text {er }}$ ou de $2^{\mathrm{e}}$ cycle.

Afin de décrire le profil des activités pharmaceutiques, nous avons calculé, pour chaque variable, le nombre total applicable par fonction à l'horaire et par année, et pour l'ensemble de ces fonctions par année également. Par souci de simplification de la présentation des résultats, les 66 fonctions à l'horaire ont été regroupées en 13 fonctions types comme suit (par ordre alphabétique) : autres fonctions, centre d'information, chirurgie, comités, enseignement, gestion, hémato-oncologie, obstétriquegynécologie, pédiatrie, services/préparations, services/validation (distribution), soins intensifs néonataux, soins intensifs pédiatriques.

Pour présenter et comparer les indicateurs de performance utilisés dans la gestion du département, nous avons calculé des ratios par fonction type de travail. Chaque ratio cible une variable à l'étude décrivant l'activité pharmaceutique divisée par le nombre total d'heures travaillées par an pour chaque fonction type. Les ratios portent sur : 1) le nombre de patients suivis par heure travaillée, 2) le nombre de demandes d'information par heure travaillée, 3) le nombre d'interventions pharmaceutiques par heure travaillée, 4) le nombre de jours-présence d'étudiants par 1816 heures travaillées. Ces variables ont été sélectionnées parce qu'ils représentent relativement bien les principales activités quotidiennes d'un pharmacien hospitalier, à savoir suivre les patients, répondre aux demandes d'information, réaliser des interventions pharmaceutiques et enseigner. Ces ratios ont été calculés par année et par fonction type.

Nous avons uniquement établi des statistiques descriptives.

\section{RÉSULTATS}

Le tableau 1 présente la comparaison des données collectées dans les différentes versions du journal de bord des pharmaciens de 2004 à 2013. Les changements apportés au contenu du journal de bord découlent de discussions entre les pharmaciens, de la volonté de refléter avec plus de justesse la charge de travail et de favoriser sa tenue au quotidien.

Pour ce qui est de l'objectif principal et du profil des activités pharmaceutiques au sein d'un établissement de santé, le tableau 2 présente le bilan des données extraites du journal 
This single copy is for your personal, non-commercial use only.

For permission to reprint multiple copies or to order presentation-ready copies for distribution, contact CJHP at cjhpedit@cshp.ca

\section{Tableau 1. Comparaison des données collectées selon les différentes versions du journal de bord des pharmaciens de 2004 à 2013*}

Catégorie des donnéest

Donnée collectée

\begin{tabular}{l}
\hline Outil \\
\hline Fonctions \\
\hline Répartition des heures \\
de travail \\
(nombre d'heures) \\
Demandes d'information \\
(nombre)
\end{tabular}

Interventions (nombre)

Version en ligne

Fonction

Services

Soins

Enseignement donné

Enseignement reçu

Recherche

Gestion

Demandes d'information

Demandes d'information interne

Demandes d'information externe

Pharmacovigilance

Continuité des soins

Conseils patients ou historique

Historique médicamenteux

Conseil au patient

BCM admission

BCM départ

BCM transfert

Pharmacocinétique, pharmacodynamique

Pharmacocinétique/génomique

Modification de thérapie

Prescription, initiation, ajustement de la thérapie

Interaction

Autres interventions

Autres activités clinico-administratives

Intervention décrite au dossier

Bon coup

Divergences involontaires résolues

Prescription d'analyse de laboratoire

Participation à la tournée

Prestation sécuritaire de soins

Étudiants (nombre de

Étudiants ou résidents

jours-présence

d'encadrement d'étudiants)

Patients ( nombre)

Suivis de patients externes

Suivis de patients internes

BCM = bilan comparatif des médicaments.

* Remarque : D'autres données collectées avant 2004 ne figurent pas dans ce tableau parce qu'elles ont été supprimées ou modifiées dans les versions présentées ici. Il s'agit du nombre d'heures travaillées sur les projets et en réunion, du degré de priorité atteint, des demandes verbales et écrites, des interventions verbales, du nombre de nouveaux patients hospitalisés et en ambulatoire suivis par le pharmacien.

tLe type de données collectées est indiquée en parenthèses (si applicable).

¥Gris clair = critères exclus de la nouvelle version, gris moyen = critères présents dans les deux versions,

gris foncé = critères introduits dans la dernière version, absence de couleur = critères absents de la version présentée.

de bord des pharmaciens, de 2004 à 2013. Les données globales recueillies pendant ces 10 ans font état de : 541448 heures travaillées, 354191 demandes d'informations, 459562 interventions pharmaceutiques, 541428 suivis de patients et 15840 jours-présence d'étudiants encadrés.

En ce qui concerne l'objectif secondaire, la présentation et la comparaison des indicateurs de performance utilisés dans la gestion du département de pharmacie, les tableaux 3 à 6 présentent les profils des quatre ratios sélectionnés selon les fonctions types de travail.
Le tableau 3 présente le profil du nombre de suivis de patients par heure travaillée selon les fonctions types. Ce ratio est passé de 0,89 en 2004 à 1,16 en 2013. En 2013, ce ratio est plus élevé dans les secteurs d'obstétrique-gynécologie (jusqu'à 70 lits d'obstétrique-gynécologie occupés en même temps) et de chirurgie (jusqu'à 45 lits de chirurgie). Les ratios sont moins élevés en soins intensifs néonataux et pédiatriques.

Le tableau 4 présente le profil du nombre de demandes d'information par heure travaillée selon les fonctions types. Ce ratio est passé de 0,60 en 2004 à 0,78 en 2013. En 2013, ce ratio 
This single copy is for your personal, non-commercial use only.

For permission to reprint multiple copies or to order presentation-ready copies for distribution, contact CJHP at cjhpedit@cshp.ca

Tableau 2. Bilan des données sur 10 ans extraites du journal de bord des pharmaciens

\begin{tabular}{|c|c|c|c|c|c|c|c|c|c|c|c|c|c|c|}
\hline Domaine & Variable & 2004 & 2005 & 2006 & 2007 & 2008 & 2009 & 2010 & 2011 & 2012 & 2013 & Somme* & * Moyenne & $\begin{array}{l}\text { Écart } \\
\text { type }\end{array}$ \\
\hline \multirow[t]{2}{*}{$\begin{array}{l}\text { Tenue du } \\
\text { journal }\end{array}$} & $\begin{array}{l}\text { Nombre } \\
\text { théorique total } \\
\text { de jours de } \\
\text { tenue du JB }(n)\end{array}$ & 6874 & 6389 & 6007 & 6554 & 6546 & 7274 & 8168 & 8319 & 7880 & 7600 & 71611 & 7161 & 805 \\
\hline & $\begin{array}{l}\text { Nombre réel } \\
\text { total de jours } \\
\text { où le JB a été } \\
\text { tenu }(n, \%)\end{array}$ & $\begin{array}{r}6732 \\
(98 \%)\end{array}$ & $\begin{array}{r}6329 \\
(99 \%)\end{array}$ & $\begin{array}{c}5922 \\
(99 \%)\end{array}$ & $\begin{array}{r}6277 \\
(96 \%)\end{array}$ & $\begin{array}{r}6439 \\
(98 \%)\end{array}$ & $\begin{array}{r}6881 \\
(95 \%)\end{array}$ & $\begin{array}{r}7453 \\
(91 \%)\end{array}$ & $\begin{array}{r}7543 \\
(91 \%)\end{array}$ & $\begin{array}{r}7187 \\
(91 \%)\end{array}$ & $\begin{array}{r}6870 \\
(90 \%)\end{array}$ & $\begin{array}{l}67633 \\
(94 \%)\end{array}$ & $\begin{array}{r}6763 \\
(94 \%)\end{array}$ & $\begin{array}{r}530 \\
(4 \%)\end{array}$ \\
\hline $\begin{array}{l}\text { Répartition } \\
\text { selon les }\end{array}$ & $\begin{array}{l}\text { Enseignement } \\
\text { donné }(h)\end{array}$ & 2152 & 2050 & 2113 & 2472 & 2205 & 2919 & 3445 & 3495 & 3197 & 3002 & 27049 & 2705 & 571 \\
\hline \multirow[t]{7}{*}{5 axes } & $\begin{array}{l}\text { Enseignement } \\
\text { reçu (h) }\end{array}$ & 1599 & 1075 & 660 & 1153 & 1190 & 1553 & 1757 & 1656 & 1353 & 992 & 12986 & 1299 & 346 \\
\hline & Services (h) & 19034 & 19363 & 20798 & 19403 & 25922 & 25294 & 24761 & 24321 & 25291 & 22091 & 226277 & 22628 & 2790 \\
\hline & Soins (h) & 23952 & 15986 & 16611 & 16678 & 17588 & 20552 & 23345 & 23463 & 22562 & 21267 & 202005 & 20200 & 3186 \\
\hline & Gestion (h) & 5901 & 5363 & 5039 & 5518 & 5111 & 5999 & 5459 & 6620 & 6542 & 6642 & 58194 & 5819 & 616 \\
\hline & Recherche (h) & 1196 & 1661 & 1219 & 995 & 1176 & 1420 & 1641 & 2499 & 1781 & 1350 & 14937 & 1494 & 432 \\
\hline & $\begin{array}{l}\text { Total des } 5 \\
\text { axes }(h)^{*}\end{array}$ & 53834 & 45498 & 46440 & 46219 & 53191 & 57736 & 60408 & 62054 & 60725 & 55343 & 541448 & 54145 & 6302 \\
\hline & $\begin{array}{l}\text { Ratio services/ } \\
\text { soins }\end{array}$ & 0,79 & 1,21 & 1,25 & 1,16 & 1,47 & 1,23 & 1,06 & 1,04 & 1,12 & 1,04 & NA & 1,14 & 0,18 \\
\hline \multirow[t]{4}{*}{ Patients } & $\begin{array}{l}\text { Suivis de } \\
\text { patients } \\
\text { internes }(n)\end{array}$ & 42785 & 35011 & 37641 & 36813 & 35939 & 46747 & 51573 & 59439 & 53654 & 51100 & 450702 & 45070 & 8659 \\
\hline & $\begin{array}{l}\text { Suivis de } \\
\text { patients } \\
\text { externes }(n)\end{array}$ & 5082 & 4704 & 5420 & 5143 & 7573 & 13518 & 12672 & 11948 & 11768 & 12898 & 90726 & 9073 & 3785 \\
\hline & $\begin{array}{l}\text { Total des suivis } \\
\text { de patients }(n)\end{array}$ & 47867 & 39715 & 43061 & 41956 & 43512 & 60265 & 64245 & 71387 & 65422 & 63998 & 541428 & 54146 & 11985 \\
\hline & $\begin{array}{l}\text { Nombre de } \\
\text { suivis de } \\
\text { patients par } \\
\text { heure travaillée } \\
(n / h)\end{array}$ & 0,89 & 0,87 & 0,93 & 0,91 & 0,82 & 1,04 & 1,06 & 1,15 & 1,08 & 1,16 & NA & 0,99 & 0,12 \\
\hline \multirow[t]{2}{*}{ Information } & $\begin{array}{l}\text { Demandes } \\
\text { d'information } \\
(n)\end{array}$ & 32496 & 30754 & 27498 & 29066 & 28255 & 30746 & 39629 & 48146 & 44636 & 42965 & \multirow{2}{*}{$\begin{array}{r}354191 \\
\text { NA }\end{array}$} & 35419 & 7661 \\
\hline & $\begin{array}{l}\text { Nombre de } \\
\text { demandes } \\
\text { d'information } \\
\text { par heure } \\
\text { travaillée }(n / h)\end{array}$ & 0,60 & 0,68 & 0,59 & 0,63 & 0,53 & 0,53 & 0,66 & 0,78 & 0,74 & 0,78 & & 0,65 & 0,09 \\
\hline
\end{tabular}

suite à la page 487

est plus élevé au centre d'information, en soins intensifs néonataux, en hémato-oncologie et aux services pharmaceutiques.

Le tableau 5 présente le profil du nombre d'interventions pharmaceutiques par heure travaillée selon les fonctions types. Ce ratio est passé de 0,64 en 2004 à 1,02 en 2013. En 2013, ce ratio est plus élevé en soins intensifs pédiatriques, en hémato-oncologie, en obstétrique-gynécologie et en soins intensifs néonataux.

Enfin, le tableau 6 présente le profil du nombre de joursprésence d'étudiants par 1816 heures travaillées selon les fonctions types. Le pharmacien employé à temps complet travaille 1816 heures par année. Ce ratio est passé de 30,97 en 2004 à 61,53 en 2013. En 2013, ce ratio est plus élevé en obstétriquegynécologie, en pédiatrie et au centre d'information.

\section{DISCUSSION}

Il existe peu de départements de pharmacie d'établissements de santé québécois qui possèdent un profil détaillé de la mesure de la charge de travail au sein de leur département depuis 1998. Cette étude descriptive présente le profil de dix années de travail pharmaceutique en établissement de santé.

Létude met en évidence la répartition des heures de travail par axe de pratique (services, soins, gestion, environnement, recherche) similaire d'année en année, peu importe la dotation en personnel. On peut établir un parallèle intéressant entre les proportions de temps accordé aux cinq axes pendant l'année 2013 au CHU Sainte-Justine (c.-à-d. $38 \%$ de soins, $40 \%$ de services, $12 \%$ de gestion, $7 \%$ d'enseignement et $3 \%$ de recherche) et les proportions dans l'enquête canadienne sur 
This single copy is for your personal, non-commercial use only.

For permission to reprint multiple copies or to order presentation-ready copies for distribution, contact CJHP at cjhpedit@cshp.ca

Tableau 2 (suite en fin). Bilan des données sur 10 ans extraites du journal de bord des pharmaciens

\begin{tabular}{|c|c|c|c|c|c|c|c|c|c|c|c|c|c|c|}
\hline Domaine & Variable & 2004 & 2005 & 2006 & 2007 & 2008 & 2009 & 2010 & 2011 & 2012 & 2013 & Somme* & Moyenne & $\begin{array}{l}\text { Écart } \\
\text { type }\end{array}$ \\
\hline \multirow[t]{10}{*}{ Interventions } & $\begin{array}{l}\text { Ajustements } \\
\text { thérapeutiques } \\
(n)\end{array}$ & 18013 & 16788 & 18382 & 19932 & 21930 & 22865 & 27548 & 26306 & 23032 & 21774 & 216570 & 21657 & 3503 \\
\hline & $\begin{array}{l}\text { Continuités } \\
\text { des soins }(n)\end{array}$ & 3228 & 3128 & 3308 & 4522 & 4726 & 10387 & 17442 & 16715 & 15001 & 14238 & 92695 & 9270 & 6091 \\
\hline & $\begin{array}{l}\text { Conseils/ } \\
\text { historiques } \\
\text { médicamenteux } \\
\text { (n) }\end{array}$ & $\begin{array}{r}4807 \\
\times\end{array}$ & 4404 & 4815 & 5160 & 5048 & 5637 & 7580 & 9284 & 7503 & 7916 & 62154 & 6215 & 1695 \\
\hline & Interactions $(n)$ & 446 & 322 & 216 & 234 & 294 & 312 & 504 & 606 & 593 & 597 & 4124 & 412 & 155 \\
\hline & $\begin{array}{l}\text { Pharmaco- } \\
\text { cinétique }(n)\end{array}$ & 2144 & 2007 & 2027 & 2149 & 1827 & 2769 & 2589 & 3129 & 3021 & 2907 & 24569 & 2457 & 479 \\
\hline & $\begin{array}{l}\text { Pharmaco- } \\
\text { vigilance } n \text { ) }\end{array}$ & 1640 & 1247 & 1422 & 2019 & 2438 & 1923 & 2640 & 2811 & 3447 & 4281 & 23868 & 2387 & 948 \\
\hline & $\begin{array}{l}\text { Autres } \\
\text { interventions }(n)\end{array}$ & 4373 & 3473 & 3018 & 2489 & 1896 & 3504 & 3977 & 3913 & 4295 & 4644 & 35582 & 3558 & 875 \\
\hline & $\begin{array}{l}\text { Total des } \\
\text { interventions }(n)\end{array}$ & 34651 & 31369 & 33188 & 36505 & 38159 & 47397 & 62280 & 62764 & 56892 & 56357 & 459562 & 45956 & 12616 \\
\hline & $\begin{array}{l}\text { Interventions } \\
\text { écrites }(n, \%)\end{array}$ & $\begin{array}{r}7557 \\
(22 \%)\end{array}$ & $\begin{array}{r}8042 \\
(26 \%)\end{array}$ & $\begin{array}{r}7187 \\
(22 \%)\end{array}$ & $\begin{array}{r}7639 \\
(21 \%)\end{array}$ & $\begin{array}{r}6664 \\
(17 \%)\end{array}$ & $\begin{array}{r}9489 \\
(20 \%)\end{array}$ & $\begin{array}{l}11614 \\
(19 \%)\end{array}$ & $\begin{array}{l}14004 \\
(22 \%)\end{array}$ & $\begin{array}{l}13379 \\
(24 \%)\end{array}$ & $\begin{array}{l}11846 \\
(21 \%)\end{array}$ & $\begin{array}{l}97421 \\
(21 \%)\end{array}$ & $\begin{array}{r}9742 \\
(21 \%)\end{array}$ & $\begin{array}{l}2738 \\
(2 \%)\end{array}$ \\
\hline & $\begin{array}{l}\text { Nombre } \\
\text { d'interventions } \\
\text { par heure } \\
\text { travaillée }(n / h)\end{array}$ & 0,64 & 0,69 & 0,71 & 0,79 & 0,72 & 0,82 & 1,03 & 1,01 & 0,94 & 1,02 & NA & 0,84 & 0,15 \\
\hline \multirow[t]{2}{*}{ Étudiants } & $\begin{array}{l}\text { Jours-présence } \\
\text { d'étudiants (JP) }\end{array}$ & 918 & 1126 & 1009 & 1240 & 1404 & 1675 & 2009 & 2462 & 2122 & 1875 & 15840 & 1584 & 524 \\
\hline & $\begin{array}{l}\text { Nombre de } \\
\text { jours } \\
\text { d'étudiants par } \\
1816 \mathrm{~h} \\
\text { travailléest } \\
\text { (JP/1816 h } \\
\text { travaillées) }\end{array}$ & 30,97 & 44,94 & 39,46 & 48,72 & 47,93 & 52,69 & 60,39 & 72,05 & 63,46 & 61,53 & NA & 52,21 & 12,34 \\
\hline
\end{tabular}

$\mathrm{JB}=$ journal de bord, $\mathrm{JP}=$ jour-présence, $\mathrm{NA}$ = non applicable.

*Données calculées à partir des valeurs exactes extraites du journal de bord. II peut y avoir un écart entre ces données et la somme des valeurs arrondies dans ce tableau.

†Remarque : Un équivalent temps plein pharmacien correspond à 1816 heures par année.

Tableau 3. Profil du nombre de suivis de patients par heure travaillée selon les fonctions types*

\begin{tabular}{|c|c|c|c|c|c|c|c|c|c|c|c|}
\hline \multirow[b]{2}{*}{$\begin{array}{l}\text { Fonctions } \\
\text { regroupées }\end{array}$} & \multicolumn{11}{|c|}{ Nombre de suivis de patients par heure travaillée } \\
\hline & 2004 & 2005 & 2006 & 2007 & 2008 & 2009 & 2010 & 2011 & 2012 & 2013 & $\begin{array}{l}\text { Moyenne } \pm \\
\text { écart type }\end{array}$ \\
\hline Chirurgie & 1,12 & 1,15 & 1,18 & 1,28 & 1,33 & 1,40 & 1,14 & 3,29 & 3,71 & 4,38 & $2,00 \pm 1,27$ \\
\hline Hémato-oncologie & 0,98 & 0,79 & 1,05 & 1,04 & 1,50 & 2,45 & 1,82 & 1,99 & 1,81 & 2,00 & $1,54 \pm 0,55$ \\
\hline $\begin{array}{l}\text { Obstétrique- } \\
\text { gynécologie }\end{array}$ & 6,68 & 5,64 & 6,83 & 5,28 & 6,25 & 6,45 & 5,53 & 5,75 & 5,11 & 4,75 & $5,83 \pm 0,70$ \\
\hline Pédiatrie & 2,27 & 2,14 & 2,40 & 2,30 & 2,26 & 2,04 & 2,03 & 2,49 & 2,36 & 2,41 & $2,27 \pm 0,16$ \\
\hline $\begin{array}{l}\text { Soins intensifs } \\
\text { néonataux }\end{array}$ & 0,62 & 1,98 & 1,95 & 2,01 & 1,62 & 1,81 & 2,15 & 2,80 & 2,12 & 1,95 & $1,90 \pm 0,54$ \\
\hline Soins intensifs & 1,59 & 1,63 & 0,79 & 1,07 & 1,32 & 1,41 & 1,04 & 0,91 & 0,93 & 0,44 & $1,11 \pm 0,37$ \\
\hline
\end{tabular}

*Fonctions retirées du tableau car inapplicables : autres fonctions, centre d'information, comités, enseignement, gestion, services/préparations, services/validation.

la pharmacie hospitalière pour l'année 2013-2014 (c.-à-d. $51 \%$ de soins, $36 \%$ de services, $6 \%$ d'autres activités, $6 \%$ d'enseignement et $1 \%$ de recherche) ${ }^{14}$. En dépit des investissements effectués dans le circuit du médicament (p. ex. informatisa- tion, robotisation, recours aux codes-barres), la proportion de temps pharmacien dévolue aux services pharmaceutiques ne diminue pas dans notre établissement (c.-à-d. $35 \%$ en 2004 c. $40 \%$ en 2013), contrairement aux résultats de l'enquête 
This single copy is for your personal, non-commercial use only.

For permission to reprint multiple copies or to order presentation-ready copies for distribution, contact CJHP at cjhpedit@cshp.ca

Tableau 4. Profil du nombre de demandes d'information par heure travaillée selon les fonctions types

\begin{tabular}{|c|c|c|c|c|c|c|c|c|c|c|c|}
\hline \multirow[b]{2}{*}{$\begin{array}{l}\text { Fonctions } \\
\text { regroupées }\end{array}$} & \multicolumn{11}{|c|}{ Nombre de demandes d'information par heure travaillée } \\
\hline & 2004 & 2005 & 2006 & 2007 & 2008 & 2009 & 2010 & 2011 & 2012 & 2013 & $\begin{array}{l}\text { Moyenne } \pm \\
\text { écart type }\end{array}$ \\
\hline Autres fonctions & 0,11 & 0,10 & 0,14 & 0,25 & 0,09 & 0,07 & 0,23 & 0,08 & 0,09 & 0,11 & $0,13 \pm 0,06$ \\
\hline $\begin{array}{l}\text { Centre } \\
\text { d'information }\end{array}$ & 1,89 & 2,10 & 1,19 & 1,31 & 1,37 & 1,47 & 1,66 & 1,44 & 1,30 & 1,29 & $1,50 \pm 0,29$ \\
\hline Chirurgie & 0,00 & 0,00 & 0,00 & 0,00 & 0,00 & 0,00 & 0,20 & 0,45 & 0,40 & 0,00 & $0,11 \pm 0,18$ \\
\hline Comités & 0,15 & 0,02 & 0,01 & 0,01 & 0,07 & 0,02 & 0,01 & 0,03 & 0,04 & 0,00 & $0,04 \pm 0,04$ \\
\hline Enseignement & 0,05 & 0,05 & 0,05 & 0,05 & 0,04 & 0,03 & 0,05 & 0,02 & 0,04 & 0,04 & $0,04 \pm 0,01$ \\
\hline Gestion & 0,32 & 0,34 & 0,30 & 0,31 & 0,31 & 0,31 & 0,35 & 0,44 & 0,42 & 0,44 & $0,35 \pm 0,06$ \\
\hline Hémato-oncologie & 0,83 & 0,74 & 0,79 & 0,87 & 0,75 & 0,88 & 0,84 & 1,06 & 1,02 & 1,13 & $0,89 \pm 0,13$ \\
\hline $\begin{array}{l}\text { Obstétrique- } \\
\text { gynécologie }\end{array}$ & 0,73 & 0,69 & 0,73 & 0,65 & 0,72 & 0,74 & 0,70 & 0,80 & 0,75 & 0,81 & $0,73 \pm 0,05$ \\
\hline Pédiatrie & 0,37 & 0,40 & 0,37 & 0,40 & 0,42 & 0,33 & 0,39 & 0,54 & 0,44 & 0,45 & $0,41 \pm 0,06$ \\
\hline $\begin{array}{l}\text { Services/ } \\
\text { préparations }\end{array}$ & 1,01 & 1,06 & 0,73 & 0,92 & 0,85 & 0,93 & 0,93 & 0,92 & 0,91 & 1,10 & $0,94 \pm 0,10$ \\
\hline Services/validation & 0,87 & 0,67 & 0,61 & 0,65 & 0,45 & 0,47 & 0,65 & 0,81 & 0,71 & 0,79 & $0,67 \pm 0,14$ \\
\hline $\begin{array}{l}\text { Soins intensifs } \\
\text { néonataux }\end{array}$ & 0,22 & 1,10 & 1,09 & 0,95 & 0,72 & 0,69 & 0,93 & 1,39 & 0,87 & 1,18 & $0,91 \pm 0,32$ \\
\hline $\begin{array}{l}\text { Soins intensifs } \\
\text { pédiatriques }\end{array}$ & 1,08 & 1,25 & 0,55 & 0,55 & 0,39 & 0,43 & 0,70 & 0,92 & 1,25 & 1,00 & $0,81 \pm 0,33$ \\
\hline
\end{tabular}

Tableau 5. Profil du nombre d'interventions pharmaceutiques par heure travaillée selon les fonctions types*

\begin{tabular}{|c|c|c|c|c|c|c|c|c|c|c|c|}
\hline \multirow[b]{2}{*}{$\begin{array}{l}\text { Fonctions } \\
\text { regroupées }\end{array}$} & \multicolumn{11}{|c|}{ Nombre d'interventions par heure travaillée } \\
\hline & 2004 & 2005 & 2006 & 2007 & 2008 & 2009 & 2010 & 2011 & 2012 & 2013 & $\begin{array}{l}\text { Moyenne } \pm \\
\text { écart type }\end{array}$ \\
\hline $\begin{array}{l}\text { Centre } \\
\text { d'information }\end{array}$ & 0,22 & 0,33 & 0,03 & 0,13 & 0,11 & 0,10 & 0,34 & 0,45 & 0,15 & 0,18 & $0,20 \pm 0,13$ \\
\hline Chirurgie & 2,53 & 2,57 & 2,81 & 3,39 & 3,63 & 3,43 & 2,55 & 1,93 & 2,24 & 1,06 & $2,61 \pm 0,77$ \\
\hline Gestion & 0,13 & 0,13 & 0,13 & 0,12 & 0,12 & 0,35 & 0,45 & 0,35 & 0,40 & 0,44 & $0,26 \pm 0,15$ \\
\hline Hémato-oncologie & 1,33 & 1,13 & 1,46 & 1,45 & 1,72 & 2,43 & 2,04 & 2,38 & 2,10 & 2,38 & $1,84 \pm 0,48$ \\
\hline $\begin{array}{l}\text { Obstétrique- } \\
\text { gynécologie }\end{array}$ & 1,78 & 1,79 & 1,66 & 1,66 & 1,80 & 2,03 & 2,19 & 2,51 & 2,10 & 1,90 & $1,94 \pm 0,27$ \\
\hline Pédiatrie & 0,70 & 0,67 & 0,65 & 0,67 & 0,65 & 0,53 & 0,53 & 0,74 & 0,68 & 0,84 & $0,67 \pm 0,09$ \\
\hline $\begin{array}{l}\text { Services/ } \\
\text { préparations }\end{array}$ & 0,27 & 0,35 & 0,48 & 0,57 & 0,58 & 0,56 & 0,53 & 0,45 & 0,34 & 0,38 & $0,45 \pm 0,11$ \\
\hline Services/validation & 0,42 & 0,30 & 0,26 & 0,23 & 0,17 & 0,16 & 0,28 & 0,32 & 0,24 & 0,30 & $0,27 \pm 0,08$ \\
\hline $\begin{array}{l}\text { Soins intensifs } \\
\text { néonataux }\end{array}$ & 0,32 & 1,55 & 1,69 & 1,64 & 1,36 & 2,06 & 3,30 & 1,80 & 1,61 & 1,73 & $1,71 \pm 0,73$ \\
\hline $\begin{array}{l}\text { Soins intensifs } \\
\text { pédiatriques }\end{array}$ & 1,73 & 1,54 & 0,79 & 2,47 & 2,06 & 1,10 & 1,93 & 2,08 & 2,76 & 2,60 & $1,91 \pm 0,64$ \\
\hline
\end{tabular}

*Fonctions retirées du tableau, car inapplicables : autres fonctions, comités, enseignement.

canadienne, où la proportion de temps consacré aux services est passée de $48 \%$ en 2003-2004 à $36 \%$ en 2013-2014, au profit des soins pharmaceutiques (c.-à-d. $38 \%$ en 2003-2004 c. $51 \%$ en 2013-2014) ${ }^{14,15}$. Plusieurs facteurs contribuent à l'importance accrue accordée aux services pharmaceutiques, notamment le cadre législatif, les exigences normatives d'Agrément Canada et de nombreux autres organismes, la complexification du circuit du médicament, l'augmentation des volumes d'activités internes, le roulement du personnel technique, les spécifications liées à la pédiatrie, comme la préparation de toutes les doses de suspension orale en seringues individuelles, etc. La tenue du journal de bord et son analyse permettent d'assurer le suivi de l'importance accordée aux différentes fonctions et aux axes de pratique en vue de l'atteinte des cibles fixées. Par exemple, un département de pharmacie qui choisirait d'augmenter la délégation de certains travaux au personnel technique afin d'accroître la couverture en soins pharmaceutiques pourrait utiliser ces données.

Si la proportion de temps consacré aux soins pharmaceutiques ne progresse pas, l'interprétation de cette donnée nécessite la prise en compte des éléments qui suivent. Au fil des années, le nombre d'équivalents temps plein au CHU SainteJustine est passé de 17 à 36 en 20 ans. Il faut aussi noter que le découpage en cinq axes de pratique que propose le journal de bord n'est pas toujours représentatif de la réalité. Par exemple, un pharmacien à la distribution (c.-à-d. fonction type 
This single copy is for your personal, non-commercial use only.

For permission to reprint multiple copies or to order presentation-ready copies for distribution, contact CJHP at cjhpedit@cshp.ca

\section{Tableau 6. Profil du nombre de jours-présence d'étudiants encadrés par 1816 heures travaillées selon les fonctions types*}

\begin{tabular}{|c|c|c|c|c|c|c|c|c|c|c|c|}
\hline \multirow[b]{2}{*}{$\begin{array}{l}\text { Fonctions } \\
\text { regroupées }\end{array}$} & \multicolumn{11}{|c|}{ Nombre de jours-présences d'étudiants par 1816 heures travailléest } \\
\hline & 2004 & 2005 & 2006 & 2007 & 2008 & 2009 & 2010 & 2011 & 2012 & 2013 & $\begin{array}{l}\text { Moyenne } \pm \\
\text { écart type }\end{array}$ \\
\hline Autres fonctions & 1,08 & 31,58 & 11,63 & 29,47 & 10,90 & 7,19 & 35,71 & 41,45 & 43,34 & 60,53 & $27,29 \pm 19,04$ \\
\hline $\begin{array}{l}\text { Centre } \\
\text { d'information }\end{array}$ & 74,78 & 182,82 & 31,32 & 63,23 & 101,67 & 157,99 & 238,64 & 172,10 & 113,58 & 96,23 & $123,23 \pm 63,38$ \\
\hline Chirurgie & 0,00 & 0,00 & 0,00 & 0,00 & 0,00 & 0,00 & 5,63 & 19,80 & 7,15 & 52,23 & $8,48 \pm 16,61$ \\
\hline Enseignement & 28,39 & 12,12 & 7,58 & 12,05 & 162,70 & 4,85 & 4,72 & 159,90 & 5,69 & 36,75 & $43,47 \pm 63,00$ \\
\hline Gestion & 26,16 & 41,35 & 58,59 & 57,48 & 62,88 & 30,66 & 35,15 & 37,72 & 57,69 & 35,68 & $44,34 \pm 13,45$ \\
\hline Hémato-oncologie & 12,04 & 15,48 & 7,86 & 25,63 & 19,68 & 46,30 & 29,42 & 42,71 & 48,39 & 44,59 & $29,21 \pm 15,35$ \\
\hline $\begin{array}{l}\text { Obstétrique- } \\
\text { gynécologie }\end{array}$ & 105,22 & 158,73 & 133,65 & 174,11 & 80,68 & 162,78 & 160,95 & 183,87 & 236,87 & 186,26 & $158,31 \pm 43,89$ \\
\hline Pédiatrie & 106,26 & 98,09 & 102,66 & 112,03 & 154,65 & 135,49 & 129,83 & 139,81 & 150,88 & 137,98 & $126,77 \pm 20,50$ \\
\hline $\begin{array}{l}\text { Services } \\
\text { préparations }\end{array}$ & 65,11 & 25,20 & 31,75 & 17,08 & 24,89 & 23,88 & 25,89 & 37,78 & 72,01 & 52,32 & $37,59 \pm 19,01$ \\
\hline Services/validation & 4,58 & 1,75 & 10,06 & 4,35 & 3,35 & 3,59 & 4,57 & 3,64 & 6,94 & 6,30 & $4,91 \pm 2,33$ \\
\hline $\begin{array}{l}\text { Soins intensifs } \\
\text { néonataux }\end{array}$ & 1,56 & 17,05 & 55,13 & 70,14 & 60,20 & 56,73 & 60,43 & 108,22 & 37,16 & 81,12 & $54,77 \pm 30,58$ \\
\hline $\begin{array}{l}\text { Soins intensifs } \\
\text { pédiatriques }\end{array}$ & 12,23 & 74,69 & 124,61 & 107,83 & 20,94 & 104,09 & 43,38 & 57,96 & 80,14 & 63,71 & $68,96 \pm 37,01$ \\
\hline
\end{tabular}

"services/validation ") qui répond à une question accomplira ses heures en services et non en soins, alors qu'il s'agit d'un élément davantage clinique, la question pouvant porter sur un patient qu'il suit régulièrement. Avec un outil plus fin permettant l'attribution des heures en plus petites unités et par activité, on aboutirait à une proportion de temps pharmacien consacrée aux soins sans doute plus élevée. En contrepartie, l'utilisation d'un tel outil au quotidien requiert beaucoup plus de temps de saisie, et l'effet net (c.-à-d. rapport entre les avantages et les inconvénients) est difficile à prévoir sans l'avoir testé.

L'étude montre qu'en 10 ans, il y a eu une augmentation de la productivité des trois ratios par heure travaillée concernant le nombre de suivis de patients (augmentation de $30 \%$ ), le nombre d'interventions (augmentation de $58 \%$ ) et le nombre de renseignements (augmentation de 29 \%). De 2004 à 2013, le ratio du nombre de jours-présence d'étudiants par 1816 heures travaillées augmente également de $99 \%$. Cette hausse de la productivité peut être attribuable à plusieurs facteurs. L'utilisation continue du journal de bord a permis d'effectuer des rétroactions individuelles ponctuelles afin d'encourager la consignation de toutes les activités au journal de bord et d'optimiser la charge de travail et les priorités. De plus, chaque secteur de soins pharmaceutiques a fait l'objet d'une révision de son offre de services dans le cadre d'une démarche structurée qui comporte une revue documentaire, une revue des volumes d'activités et une réflexion sur les changements à apporter afin d'accroître la pertinence et la quantité de soins pharmaceutiques ${ }^{16-26}$. En outre, la pénurie de pharmaciens s'est atténuée au fil du temps (c.-à-d. $17 \%$ de pénurie de pharmaciens en 2004 c. $33 \%$ en 2006 c. $12 \%$ en 2013).
Certaines variations de ratios observées s'expliquent par des événements circonscrits au cours de la période étudiée. Par exemple, le nombre d'heures de services par rapport au nombre d'heures de soins montre une prépondérance des soins pharmaceutiques en 2004 (ratio =0,79). Ce rapport s'inverse de 2005 à 2009, les services ayant été privilégiés par rapport aux soins, compte tenu de la pénurie de pharmaciens. À partir de 2010, l'équilibre entre les soins et les services se stabilise. Avec la mise en place du nouveau programme de doctorat professionnel en pharmacie (Pharm. D) en 2008, le nombre de jours-présence d'étudiants a augmenté de $51 \%$ de 2007 à 2013 par l'arrivée des étudiants de deuxième année en stage hospitalier et par l'augmentation du nombre de stagiaires de $4^{\mathrm{e}}$ année. Le bilan comparatif des médicaments a été mis en place progressivement à partir de 2007. Dès 2008, cette instauration a été suivie par une augmentation du nombre de suivis de patients hospitalisés (augmentation de $39 \%$ de 2007 à 2013), du nombre d'interventions de conseils et d'historiques médicamenteux (augmentation de $51 \%$ de 2007 à 2013) et du nombre d'interventions de continuité des soins (augmentation de $215 \%$ de 2007 à 2013). La mise en place d'un programme structuré de pharmacovigilance en 2008, avec notamment la participation au Canadian Pharmacogenomics Network for Drug Safety, est associée à une hausse du nombre d'activités de pharmacovigilance (+76\% de 2008 à 2013). En vertu d'une entente locale, puis de l'entente de travail entre le MSSS et l'Association des pharmaciens en établissement de santé du Québec (APES), la semaine de travail de tous les pharmaciens a été portée à 40 heures travaillées, comme suite à l'ajustement salarial de $20 \%$ accordé aux pharmaciens en 2007. L'augmenta- 
tion de $17 \%$ des heures totales travaillées entre 2007 et 2008 démontre bien les retombées de cette entente. Toutefois, les détails recueillis ne permettent pas de saisir toutes les activités. Par exemple, la réduction du nombre d'ajustements cinétiques de la vancomycine liée à l'élimination du suivi des pics est masquée par l'émergence de la cinétique de nouvelles molécules (p. ex. linézolide, voriconazole).

Cette étude démontre qu'il est possible d'implanter et d'utiliser de façon pérenne un journal de bord en pharmacie hospitalière. Bien que le taux de tenue du journal soit passé de $98 \%$ en 2004 à $90 \%$ en 2013, le taux de participation reste élevé. La baisse de ce taux s'explique notamment par un essoufflement de l'équipe à utiliser ce type d'outils et au fait que certains membres en voient moins la pertinence. L'outil développé en version papier puis en version électronique sur un intranet ne requiert aucun investissement important (c.-à-d. quelques heures de programmation sur une page web en asp.net). Les données saisies sont recueillies dans la base de données Structured Query Language (SQL) et des requêtes sont effectuées périodiquement afin d'établir un profil de la situation. Notre équipe envisage la possibilité, dans un avenir plus ou moins proche, d'une application mobile qui faciliterait la collecte en temps réel. L'établissement de santé ne fournit pas de téléphones portables, de sorte que l'application doit être développée dans au moins deux environnements (c.-à-d. iPhone, Android). Les coûts inhérents à ce développement et aux mises à jour ne sont pas négligeables, et une minorité de pharmaciens en souhaitent actuellement le déploiement.

Une fois développé, cet outil ne requiert que 5 à 7 minutes par jour et cible un nombre limité d'indicateurs. En 1998, dans les discussions initiales entourant la mise en place de ce journal de bord, certains types d'indicateurs ont été volontairement omis. Par exemple, le temps accordé à chaque type d'intervention n'est pas mesurable au quotidien de manière réaliste. La mesure du taux d'acceptation des interventions est dépassée, et la majorité des pharmaciens interviennent avec pertinence, de sorte que ce taux est reconnu comme étant élevé dans la littérature scientifique ${ }^{10,27}$. Il n'y a pas d'indicateurs de retombées, puisqu'elles ne sont souvent pas évaluables à court terme.

Cette étude présente un journal de bord à déclaration quotidienne. Une telle approche est pertinente parce qu'elle établit un mécanisme quotidien de responsabilisation. La littérature spécialisée rapporte aussi des cas de journaux de bord colligés par échantillonnage sur de courtes périodes (p. ex. une semaine à la fois mais de façon plus exhaustive ${ }^{28,29}$. La déclaration quotidienne s'inscrit dans les obligations de consignation exigées à plusieurs autres groupes de professionnels dans les établissements de santé québécois (p. ex. psychologues, physiothérapeutes, ergothérapeutes, travailleurs sociaux).

Le journal de bord en place a été utilisé à diverses fins. Il s'agit d'un bon outil qui montre aux cliniciens, aux adminis- trateurs, aux professeurs et aux décideurs la contribution soutenue des pharmaciens aux cinq axes de pratique. L'outil facilite la réponse aux questions de l'enquête canadienne biannuelle sur la pharmacie hospitalière. L'outil permet de montrer l'effet des pénuries sur les activités pharmaceutiques. Le journal de bord contribue à l'émulation par les pairs, bien qu'aucune donnée individuelle ne soit diffusée à moins qu'une personne souhaite obtenir une rétroaction sur ses propres données. En outre, la tenue régulière de ce journal confirme le sérieux d'une équipe professionnelle à consigner son activité au quotidien.

Cette étude s'inscrit dans le cadre des travaux de Fernandes et coll. portant sur les indicateurs clés de performance en pharmacie clinique ${ }^{13}$. Étant donné la participation de notre établissement à cette démarche et l'importance d'intégrer de nouveaux indicateurs au journal de bord, des modifications ont été apportées à ce journal en 2014. Il est toutefois nécessaire d'inclure la ventilation des heures travaillées dans les cinq axes de pratique et la consignation des activités pharmaceutiques au journal de bord départemental, quelle que soit la fonction à l'horaire. Par exemple, un pharmacien doit consigner ses interventions à la distribution ou encore un chef de département de pharmacie doit consigner le nombre de demandes d'information auxquelles il répond. Cependant, il n'existe pas encore de consensus au sein de la profession entourant l'ensemble des indicateurs de mesure de l'activité pharmaceutique sur une base réaliste et opérationnelle. Il semble, avec le temps, qu'un tel consensus ne peut émerger que si une autorité professionnelle ou gouvernementale l'exige. Néanmoins, ces travaux peuvent fournir matière à réflexion sur la mesure de la charge de travail en pharmacie hospitalière.

Dans le contexte d'un éventuel financement à l'activité des établissements de santé, il est pertinent d'établir un outil simple et cohérent de mesure de l'activité pharmaceutique pour répartir adéquatement les heures travaillées. Les données présentées dans cette étude ont notamment servi à établir la matrice de répartition des ressources humaines aux fins du financement éventuel des hôpitaux sur la base d'une tarification à l'acte.

Cette étude descriptive comporte des limites. Il s'agit de la tenue interne d'un journal, et il n'existe pas de processus formel d'audit pour confirmer la véracité des données fournies. Toutefois, la demande exprimée par l'employeur de tenir un journal de bord s'inscrit dans le cadre de l'exercice de la pharmacie et repose notamment sur les exigences du Code de déontologie des pharmaciens, dont celles qui ont trait à l'intégrité. Tous les pharmaciens ne remplissent pas obligatoirement chaque jour le journal de bord en ligne, et les données recueillies proviennent d'outils de travail personnels. Un biais de mémoire peut intervenir dans la tenue des journaux de certains pharmaciens, bien que les tendances par personne (non décrites dans cet article) ou par fonction ou fonction type sont remarquablement stables 
au cours d'une même année. En temps opportun, des rappels périodiques individualisés doivent être effectués pour améliorer le taux de réponses. L'adaptation du journal de bord aux nouveaux indicateurs clés de performance de pharmacie clinique limite la comparaison des données avant et après la mise à jour du journal de bord en 2014. Les données recueillies ne mesurent ni le taux d'acceptation ni les retombées des interventions. D'autres travaux sont nécessaires afin d'évaluer ces points. En outre, cette étude ne porte que sur un établissement. S'il est souhaitable que cet outil soit utilisé pour des comparaisons entre les établissements, il faut qu'un tiers externe (p. ex. le MSSS, l'APES) statue sur un outil pour qu'il soit utilisé à large échelle. Des travaux menés par les chefs de départements de pharmacie de cinq centres hospitaliers universitaires québécois ont mis en évidence des différences importantes en ce qui concerne les indicateurs de mesure, la faisabilité de collecte et la pertinence perçue. On peut imaginer que, sans une action concertée d'un tiers-décideur, il est peu probable que tous les établissements réussissent à s'entendre sur un seul outil de mesure reposant sur les mêmes définitions ${ }^{30}$.

\section{CONCLUSION}

Il est possible de consigner de façon cohérente et récurrente l'activité pharmaceutique au sein d'un centre hospitalier québécois à l'aide d'un journal de bord sur une période de 10 ans. Cet outil permet non seulement une mesure quotidienne et efficiente de l'activité pharmaceutique, mais il offre aussi une vue d'ensemble à l'équipe de gestion et à toute l'équipe de pharmaciens de la prestation optimale de services et de soins pharmaceutiques, tout en assurant la bonne utilisation des ressources professionnelles en pharmacie hospitalière.

\section{Références}

1. Critère 2.1.8. Dans : Standards de pratique. Montréal (QC) : Ordre des pharmaciens du Québec; 2010. Publié au : http://www.opq.org/cms/ Media/290_38_fr-CA_0_2982_standards_pratique_opq_web.pdf. Consulté le 17 mai 2016.

2. American Society of Hospital Pharmacists (ASHP). ASHP guidelines on documenting pharmaceutical care in patient medical records. Am J Health Syst Pharm. 2003;60(7):705-7.

3. Standardisation valorisation activités pharmaceutiques - interventions pharmaceutiques. Société Française de Pharmacie Clinique (SFPC); mis à jour le 11 novembre 2015. Publié au : http://sfpc.eu/fr/pratiquesprofessionelles/33-interventions-pharmaceutiques.html. Consulté le 17 mai 2016.

4. Schnell BR, Gesy KF, Gaucher ME. The development of a Canadian hospital pharmacy workload measurement system. Can J Hosp Pharm. 1981;34(3):75-8

5. Ruth S, Eagleson D, Ilersich AL. Workload measurement in long term care pharmacy. Can J Hosp Pharm. 1990;43(3):109-15.

6. Kaplan B, Lorenzo AG, Nystrom KK. Validating a clinical workload measurement instrument for documenting pharmaceutical care. Pharm Pract Manag Q. 1996;15(4):53-63.
7. Bajcar J, Chin T, Chui W, Wichman K. Development of a comprehensive clinical pharmacy workload documentation system. Can J Hosp Pharm. 1995;48(2):80-9.

8. Dagenais J, Grenier S. Key pharmacy indicators: capturing workload and measuring operational performance in a Canadian Forces clinic. Can Pharm J. 2013;146(2):88-92.

9. Collins MF. Measuring performance indicators in clinical pharmacy services with a personal digital assistant. Am J Health Syst Pharm. 2004; 61(5):498-501.

10. Lindblad AJ, Alleyne A, Howorko J. Development and initial evaluation of a software-based clinical workload measurement system for pharmacists. Can J Hosp Pharm. 2007;60(5):295-301.

11. Magarinos-Torres R, Osório-de-Castro CG, Edais Pepe VL. [Establishment of criteria and outcome indicators for hospital pharmacies in Brazil using Delphos]. Cad Saude Publica. 2007;23(8):1791-802. Article en portugais.

12. $\mathrm{Ng} \mathrm{J}$, Harrison J. Key performance indicators for clinical pharmacy services in New Zealand public hospitals: stakeholder perspectives. $J$ Pharm Health Serv Res. 2010;1(2):75-84.

13. Fernandes O, Gorman SK, Slavik RS, Semchuk WM, Shalansky S, Bussières JF, et al. Development of clinical pharmacy key performance indicators for hospital pharmacists using a modified Delphi approach. Ann Pharmacother. 2015;49(6):656-69.

14. Bonnici A, Wilgosh C. Chapitre D : Ressources humaines. Dans : Bonnici A, Bornstein C, Bussières JF, Doucette D, Hall K, Jones R, et al., comité de rédaction. Rapport sur les pharmacies hospitalières canadiennes 2013-2014. Eli Lilly; 2015. pages 40-58. Publié au : http:// stage.lillyhospitalsurvey.ca/hpc2/content/2015_report/chapter_dF\%20. pdf. Consulté le 25 mai 2016.

15. Johnson N. Ressources humaines. Dans : McKerrow R, Bussières JF, Johnson N, Macgregor P, Hall KW, Lefebvre P, et al., comité de rédaction. Rapport annuel 2003-2004. Pharmacies hospitalières au Canada. Prestation sécuritaire de soins et utilisation des médicaments. Eli Lilly; 2005. pages 50-8. Publié au : http://stage.lillyhospitalsurvey.ca/hpc2/content/2004_ Report/f50.pdf. Consulté le 25 mai 2016.

16. Guérin $\mathrm{A}$, Thibault $\mathrm{M}$, Nguyen $\mathrm{C}$, Lebel D, Bussières JF. Démarche pour la mise à niveau de soins pharmaceutiques : le cas de la nutrition parentérale. J Pharm Clin. 2015;34(3):147-59.

17. Leroux A, Guérin A, Bussières JF, Lebel D, Tremblay S, Roy H, et al. Mise à niveau d'un secteur pédiatrique de soins pharmaceutiques au Québec. Arch Pediatr. 2016;23(2):117-27.

18. Stöckel F, Bédard P, Métras ME, Bussières JF. Mise à jour des activités pharmaceutiques dans une clinique externe de VIH/SIDA pédiatrique. Pharm Hosp Clin. 2015;50(4):422-33.

19. Guérin A, Rivaille F, Bussières JF. Les grossistes-répartiteurs : comparaison Québec/France. Actual Pharm. 2015;543:36-40.

20. Guérin A, Bédard P, Lebel D, Bussières JF. Démarche pour la mise à niveau de soins pharmaceutiques en établissement de santé : l'exemple de l'immunisation. Santé Publique. 2015;26(6):813-28.

21. Guérin A, Caron E, Ouellette Frève JF, Lebel D, Bussières JF. Mise à niveau d'un secteur de soins pharmaceutiques : le cas de la fibrose kystique. Can J Hosp Pharm. 2014;67(3):230-9.

22. Guérin A, Thibault M, Nguyen C, Lebel D, Bussières JF. Démarche pour la mise à niveau d'un secteur de soins pharmaceutiques : le cas de la chirurgie pédiatrique. Ann Pharm Fr. 2014;72(4):217-302.

23. Bussières JF, Tollec S, Martin B, Malo J, Tardif L, Thibault M. Démarche pour la mise à niveau d'un secteur de soins pharmaceutiques : le cas de la néonatologie. Ann Pharm Fr. 2010;68(3):178-94.

24. Touzin K, Robelet A, Therrien R, Bussières JF. Démarche pour la mise à niveau d'un secteur de soins pharmaceutiques : le cas de l'hématooncologie. Can J Hosp Pharm. 2010;63(2):119-29.

25. Robelet A, Bussières JF, Boivin J, Garel P. Évaluation des besoins pharmacothérapeutiques d'un service de pédopsychiatrie. Neuropsychiatr Enfance Adolesc. 2008;56:439-45.

26. Robelet A, Bussières JF, Marquis C, Lavoie A, Lebel D. Description d'une démarche de mise à niveau de prestation de pharmacie clinique en réanimation pédiatrique. J Pharm Clin. 2007;26(4):217-27. 
27. Guignard B, Bonnabry P, Perrier A, Dayer P, Desmeules J, Samer CF. Drug-related problems identification in general internal medicine: the impact and role of the clinical pharmacist and pharmacologist. Eur J Intern Med. 2015;26(6):399-406.

28. James KL, Barlow D, Bithell A, Burfield R, Hiom S, Lord S, et al. Measuring dispensary workload: a comparison of the event recording and direct time techniques. Int J Pharm Pract. 2011;19(4):264-75.

29. Penaforte TR, Forste AC, Simões MJ. Evaluation of the performance of pharmacists in terms of providing health assistance at a university hospital. Clinics (Sao Paulo). 2007;62(5):567-72.

30. Bussières JF, Lefebvre $\mathrm{P}$, Bois $\mathrm{D}$, Vallée $\mathrm{M}$, Théberge $\mathrm{M}$, Racine MC. Indicateurs et pratique pharmaceutique: Le point de vue des cinq centres hospitaliers universitaires du Québec. Pharmactuel. 2010;43(2):117-28.

Sophie Dubois est une assistante de recherche, Unité de recherche en pratique pharmaceutique et Département de pharmacie, Centre hospitalier universitaire Saint-Justine, Montréal (Québec). Elle est aussi une étudiante dans le programme D. Pharm dans la Faculté des sciences pharmaceutiques et biologiques de Rennes, Université de Rennes 1 , Rennes, France.
Denis Lebel, B. Pharm., M. Sc., FCSHP, est adjoint au chef de département, Unité de recherche en pratique pharmaceutique et Département de pharmacie, Centre hospitalier universitaire Saint-Justine, Montréal (Québec).

Jean-François Bussières, B. Pharm., M. Sc., MBA, FCSHP, est chef, Unité de recherche en pratique pharmaceutique et Département de pharmacie, Centre hospitalier universitaire Saint-Justine, et professeur titulaire de clinique, Faculté de pharmacie, Université de Montréal, Montréal (Québec)

Intérêts concurrents : Aucun déclaré.

\section{Adresse pour correspondance :}

Jean-François Bussières

Département de pharmacie

Centre hospitalier universitaire Sainte-Justine

3175, chemin de la Côte Sainte-Catherine

Montréal QC H3T 1C5

Courriel : jf.bussieres@ssss.gouv.qc.ca

Financement : Aucun reçu.

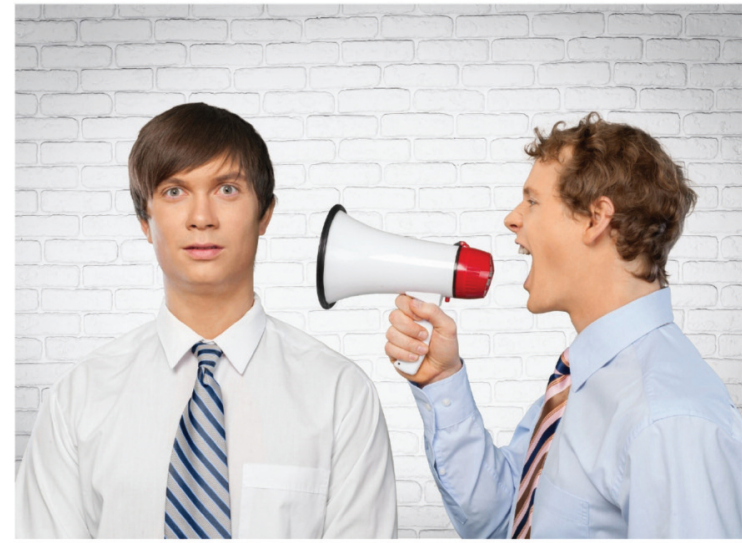

REFER A FRIEND
$\mathrm{D}$

you know that most of our new members and supporters learn about CSHP through word of mouth?

Over the years, you have referred hundreds of your friends and colleagues to the Society-THANK YOU!

We'd like to encourage you to keep talking about us!

CSHP offers a referral program that allows you to gain credit that can be applied to publications, continuing education programs, and membership.

* Receive a $\mathbf{\$ 1 0}$ credit for referring other members and supporters.

* Receive a $\mathbf{\$} 5$ credit for referring other students and residents.

New members and supporters will have the opportunity to list you as the person who referred them to CSHP on their application-it's that simple!

For more information, please contact:

Membership Services

Telephone: (613) 736-9733, ext. 222

Email: membershipservices@cshp.ca 